\title{
Lowness and the Complexity of Sparse and Tally Descriptions
}

\author{
V. Arvind* \\ Department of Computer Science and Engineering \\ Indian Institute of Technology, Delhi \\ New Delhi 110016, India \\ J. Köbler and M. Mundhenk \\ Abteilung für Theoretische Informatik \\ Universität Ulm, Oberer Eselsberg \\ D-W-7900 Ulm, Germany
}

\begin{abstract}
We investigate the complexity of obtaining sparse descriptions for sets in various reduction classes to sparse sets. Let $A$ be a set in a certain reduction class $R_{r}$ (SPARSE). Then we are interested in finding upper bounds for the complexity (relative to $A$ ) of sparse sets $S$ such that $A \in R_{r}(S)$. By establishing such upper bounds we are able to derive the lowness of $A$.

In particular, we show that if a set $A$ is in the class $R_{h d}^{p}\left(R_{c}^{p}\right.$ (SPARSE)) then $A$ is in $R_{h d}^{p}\left(R_{c}^{p}(S)\right)$ for a sparse set $S \in \mathrm{NP}(A)$. As a consequence we can locate $R_{h d}^{p}\left(R_{c}^{p}\right.$ (SPARSE)) in the $E L_{3}^{\Theta}$ level of the extended low hierarchy. Since $R_{h d}^{p}\left(R_{c}^{p}\right.$ (SPARSE) $) \supseteq R_{b}^{p}\left(R_{c}^{p}\right.$ (SPARSE) $)$ this solves the open problem of locating the closure of sparse sets under bounded truth-table reductions optimally in the extended low hierarchy. Furthermore, we show that for every $A \in R_{d}^{p}$ (SPARSE) there exists a sparse set $S \in \mathrm{NP}(A \oplus \mathrm{SAT}) / \mathrm{F} \Theta_{2}^{p}(A)$ such that $A \in R_{d}^{p}(S)$. Based on this we show that $R_{1-t t}^{p}\left(R_{d}^{p}(\mathrm{SPARSE})\right)$ is in $E L_{3}^{\Theta}$.

Finally, we construct for every set $A \in R_{c}^{p}$ (TALLY) $\cap R_{d}^{p}$ (TALLY) (or equivalently, $A \in \mathrm{IC}\left[\log\right.$, poly], as shown in $\left.\left[\mathrm{AHH}^{+} 92\right]\right)$ a tally set $T \in \mathrm{P}(A \oplus \mathrm{SAT})$ such that $A \in R_{c}^{p}(T) \cap R_{d}^{p}(T)$. This implies that the class IC [log, poly] of sets with low instance complexity is contained in $E L_{1}^{\Sigma}$.
\end{abstract}

\section{Introduction}

Sparse sets play a central role in structural complexity theory. The question of the existence of sparse hard sets for various complexity classes under different sorts of reducibilities is well studied (see for example [KL80, Mah82, OW91, AHH+92, AKM92]).

${ }^{*}$ Work done while visiting Universität Ulm. Supported in part by an Alexander von Humboldt research fellowship. 
Besides that much work has been done on other issues concerning the complexity of sets reducible to sparse sets (see for example [Kad87, AH92, SL92, AHH $\left.{ }^{+} 92\right]$ ). A central motivation for most earlier work (and also for this paper) can be seen as seeking answers to the following two questions.

1. If a set $A$ is reducible to a sparse set, does it follow that $A$ is reducible to some sparse set that is "simple" relative to $A$ ?

2. If a set $A$ is reducible to a sparse set, then how easy is it to access all the relevant information contained in the set $A$ when it is used as oracle?

Question 1 originates in the study of the notions of equivalence and reducibility to sparse sets (see for example [TB91, AHOW, GW91]) and addresses the complexity (relative to $A$ ) of sparse descriptions for sets $A$ which are reducible to sparse sets. For

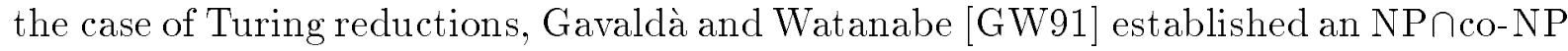
lower bound by constructing a set $B \in R_{T}^{p}$ (SPARSE) (in fact, $B$ is even in $R_{c}^{p}$ (SPARSE)) that is not Turing reducible to a sparse set in $\mathrm{NP}(B) \cap$ co- $\mathrm{NP}(B)$. For truth-table reduction classes to sparse sets, the first question is also investigated in $\left[\mathrm{AHH}^{+}\right.$92] where various upper bounds for the relative complexity of sparse descriptions are presented.

The second question concerns lowness properties of sets reducible to sparse sets. Lowness is an important structural tool for systematically classifying sets in different complexity classes according to the power they provide when used as oracles. Building on ideas from recursive function theory, Schöning [Sch83] defined the low hierarchy inside NP. In order to classify sets outside of NP (e.g. sets reducible to sparse sets) Balcázar, Book, and Schöning [BBS86] defined the extended low hierarchy. The extended low hierarchy was subsequently refined by Allender and Hemachandra [AH92] and Long and Sheu [LS91] who showed the optimal location of various reduction and equivalence classes to sparse and tally sets in the (refined) extended low hierarchy. Very recently, Sheu and Long [SL92] proved that the extended low hierarchy is an infinite hierarchy.

In order to investigate the first question, we generalize the definition of sparse set descriptions given in [GW91]. Let $\leq_{r}$ be a reducibility. A sparse set $S$ is a sparse $r$-description for a set $A$ if $A \leq_{r} S$. Similarly, a tally set $T$ is a tally $r$-description for $A$ if $A \leq_{r} T$. In particular a sparse set $S$ is called a sparse description for $A$ if $A \leq_{T}^{p} S$. We are interested in finding upper bounds for the relative complexity of sparse (tally) $r$-descriptions for sets in $R_{r}(\mathrm{SPARSE})^{1}$ (respectively $R_{r}$ (TALLY)). We refer to a sparse $r$-description satisfying the established upper bound as a simple sparse $r$-description (with respect to that upper bound), and we refer to the corresponding upper bound result as a simplicity result.

In Section 4 we establish simplicity results for various reduction classes to sparse and tally sets. In Section 5 we apply the simplicity results to derive lowness properties. These results reveal a close interconnection between the lowness of sets which are reducible to sparse or tally sets and the complexity of computing small descriptions for these sets. More precisely, the general pattern to prove the lowness of a set $A$ that reduces to a sparse set is the following: first we appropriately bound the complexity of a sparse description for $A$; based on such a simplicity result, we then apply a deterministic enumeration technique like that of Mahaney [Mah82] or a census technique similar to that of Hemachandra [Hem87] and Kadin [Kad87] to replace the sparse oracle $S$ (and

\footnotetext{
${ }^{1}$ For a reducibility $\leq_{r}$ and a class $\mathcal{C}$ of sets, $R_{r}(\mathcal{C})=\left\{A \mid A \leq_{r} B\right.$ for some $\left.B \in \mathcal{C}\right\}$.
} 
thus $A$ ). Intuitively, we use the simplicity result to extract enough information (a suitable initial segment of $S$ or the census thereof) from the oracle set $A$ in order to avoid further queries to $A$. Our approach clarifies that the appropriate simplicity result is the basic reason for the set to be low. Using this approach we are able to derive several new and optimal lowness results.

\section{Overview of results}

The main results in this paper are the following.

We show that for every set $A \in R_{h d}^{p}\left(R_{c}^{p}\right.$ (SPARSE)) there exists a sparse set $S \in \mathrm{NP}(A)$ such that $A \in R_{h d}^{p}\left(R_{c}^{p}(S)\right)$. We use this result to locate $R_{h d}^{p}\left(R_{c}^{p}\right.$ (SPARSE)) and, as a consequence, also $R_{b}^{p}\left(R_{c}^{p}\right.$ (SPARSE)) in the $E L_{3}^{\Theta}$ level of the extended low hierarchy. This solves an open problem posed in [AH92] regarding the location of $R_{2-t t}^{p}$ (SPARSE) in the extended low hierarchy. Since there exist sparse sets that are not in $E L_{2}^{\Sigma}$ [AH92] the location of $R_{h d}^{p}\left(R_{c}^{p}\right.$ (SPARSE)) in $E L_{3}^{\Theta}$ is optimal.

Furthermore, we show that for each set $A$ which reduces via a disjunctive reduction to a sparse set there exists a sparse set $S \in \mathrm{NP}(A \oplus \mathrm{SAT}) / \mathrm{F}_{2}^{p}(A)$ such that $A \in R_{d}^{p}(S)$. Based on this we show that $R_{1-t t}^{p}\left(R_{d}^{p}(\mathrm{SPARSE})\right)$ is also contained in $E L_{3}^{\Theta}$. Previously it was only known that $R_{d}^{p}$ (SPARSE) lies in $E L_{3}^{\Sigma}$ (derived from the $E L_{3}^{\Sigma}$-lowness of $\mathrm{P} /$ poly [BBS86]). (We note that very recently it has been proved that $\mathrm{P} /$ poly is contained in $E L_{3}^{\Theta}$ [Köb92] using a different and more involved proof technique.)

Next we show that for every set $A$ in $R_{m}^{c o-n p}$ (SPARSE) there exists a sparse set $S \in R_{m}^{n p}(A)$ such that $A \in R_{m}^{c 0^{-n p}}(S)$, and that for every set $A$ in $R_{h d}^{p}\left(R_{m}^{c 0^{-} n p}\right.$ (SPARSE)) there exists a sparse set $S \in \mathrm{NP}(\mathrm{SAT} \oplus A)$ such that $A \in R_{h d}^{p}\left(R_{m}^{c c^{-} n p}(S)\right)$. As a consequence we get that NP(NP $\cap$ SPARSE) $\cap R_{m}^{c o-n p}$ (SPARSE) is low for $\Theta_{2}^{p}$, and that for $A \in R_{h d}^{p}\left(R_{m}^{c o-n p}\right.$ (SPARSE)) it holds that $\Theta_{3}^{p}(A) \subseteq \Theta_{2}^{p}\left(\Sigma_{2}^{p} \oplus A\right)$. Since $R_{b}^{p}\left(R_{m}^{c 0^{-} n p}(\mathrm{SPARSE})\right) \subseteq R_{h d}^{p}\left(R_{m}^{c 0^{-n p}}(\mathrm{SPARSE})\right)$, we get similar results for the class $R_{b}^{p}\left(R_{m}^{c 0^{-n p}}(\mathrm{SPARSE})\right)$ as corollaries.

Finally we locate the class IC $[\log$, poly] of sets containing only strings of low instance complexity in the first level $E L_{1}^{\Sigma}$ of the extended low hierarchy. The simplicity result behind the lowness of IC[log, poly] (which equals $R_{c}^{p}$ (TALLY) $\cap R_{d}^{p}(\mathrm{TALLY})\left[\mathrm{AHH}^{+} 92\right]$ ) is that for every set $A \in R_{c}^{p}$ (TALLY $) \cap R_{d}^{p}$ (TALLY) there is a tally set $T$ in $\mathrm{P}(\mathrm{SAT} \oplus A)$ such that $A \in R_{c}^{p}(T) \cap R_{d}^{p}(T)$.

\section{Preliminaries and notation}

Let $A$ be a set. $\chi_{A}$ denotes the characteristic function of $A . A^{=n}\left(A^{\leq n}\right)$ denotes the set of all strings in $A$ of length $n$ (up to length $n$, respectively). The cardinality of $A$ is denoted by $|A|$. A set $T$ is called a tally set if $T \subseteq 0^{*}$. The census function of a set $A$ is census $_{A}\left(1^{n}\right)=\left|A^{\leq n}\right|$. A set $S$ is called sparse if its census function is bounded above by a polynomial. We use TALLY and SPARSE to represent the classes of tally and sparse sets, respectively.

The join of two sets $A$ and $B$, denoted $A \oplus B$, is defined as $A \oplus B=\{0 x \mid x \in$ $A\} \cup\{1 x \mid x \in B\} .\langle\cdot, \cdot\rangle$ denotes a standard polynomial time invertible pairing function such that $\left\langle 0^{i}, 0^{j}\right\rangle \in 0^{*}$ for all $i, j \geq 0$. Such a pairing function can be extended in a standard fashion to encode arbitrary finite sets of strings. For a class $\mathcal{C}$ of sets we denote 


\begin{tabular}{|l|l|}
\hline Name & Notation \\
\hline \hline many-one & $\leq_{m}^{p}$ \\
one truth-table & $\leq_{1-t t}^{p}$ \\
bounded truth-table & $\leq_{b}^{p}$ \\
conjunctive & $\leq_{c}^{p}$ \\
disjunctive & $\leq_{d}^{p}$ \\
bounded Hausdorff & $\leq_{b h d}^{p}$ \\
Hausdorff & $\leq_{h d}^{p}$ \\
$n p$ many-one & $\leq_{m}^{n p}$ \\
co-np many-one & $\leq_{m}^{c o-n p}$ \\
\hline
\end{tabular}

Table 1: Polynomial-time Reductions

the union of all sets in $\mathcal{C}$ by $\cup \mathcal{C}$. A set $S$ is called $\mathrm{P}(\mathcal{C})$-printable (see [HY84]) if the function $0^{n} \mapsto\left\langle S^{\leq n}\right\rangle$ can be computed inside $\operatorname{FP}(\mathcal{C})$.

The reducibilities discussed in this paper are the standard polynomial time bounded reducibilities defined by Ladner, Lynch, and Selman [LLS75] and the Hausdorff reduction introduced by Wagner [Wag87] (see Table 1 for an overview).

Definition 3.1 [Wag87] $A$ is Hausdorff-reducible to $B\left(A \leq_{h d}^{p} B\right)$, if there exists a function $f$ computable in polynomial time, such that for all $x, f$ computes a tuple $f(x)=\left\langle y_{1}, y_{2}, \ldots, y_{2 k}\right\rangle$ such that

1. $\chi_{B}\left(y_{1}\right) \geq \chi_{B}\left(y_{2}\right) \geq \cdots \geq \chi_{B}\left(y_{2 k}\right)$, and

2. $\chi_{A}(x)=\bigvee_{i=1}^{k}\left[\chi_{B}\left(y_{2 i-1}\right) \wedge \neg \chi_{B}\left(y_{2 i}\right)\right]$,

We call $f$ a bounded Hausdorff reduction $\left(A \leq_{b h d}^{p} B\right)$ if $k$ is a constant.

Notation [AHOW] For any reducibility $\leq_{r}^{\alpha}$ where $\alpha \in\{p, n p$, co-np $\}$ and $r \in$ $\{m, c, d, 1-t t, b, h d, b h d\}$ and any class $\mathcal{C}$ of sets let $R_{r}^{\alpha}(\mathcal{C})=\left\{A \mid A \leq_{r}^{\alpha} B\right.$ for some $B \in \mathcal{C}\}$.

A class $\mathcal{K}$ of sets that includes $\emptyset$ and $\Sigma^{*}$ and is closed under union and intersection is said to be a set ring. The following characterization of the boolean closure of set rings due to Hausdorff plays a key role in some of our results.

Theorem 3.2 [Hau14] Let $\mathcal{K}$ be a set ring and let $B C(\mathcal{K})$ be the closure of $\mathcal{K}$ under union, intersection, and complement. Then every $A \in B C(\mathcal{K})$ can be represented as $A=\bigcup_{i=1}^{k}\left(A_{2 i-1}-A_{2 i}\right)$, where $A_{j} \in \mathcal{K}, 1 \leq j \leq 2 k$, and $A_{1} \supseteq A_{2} \supseteq \cdots \supseteq A_{2 k}$.

The above representation for $A$ is called a Hausdorff representation for $A$ over $\mathcal{K}$. We state some useful properties relating boolean closures, bounded truth-table closures, and the bounded Hausdorff closures of language classes.

Lemma 3.3 [KSW87] Let $\mathcal{K}$ be a class that contains $P$ and is closed under many-one reductions. Then $B C(\mathcal{K})=R_{b}^{p}(\mathcal{K})$. 
Lemma 3.4 Let $\mathcal{K}$ be a class closed under many-one reductions and under join. Then $A$ has a Hausdorff representation over $\mathcal{K}$ if and only if $A$ is bounded Hausdorff reducible to some set in $\mathcal{K}$.

Proof Let $A=\bigcup_{i=1}^{k}\left(A_{2 i-1}-A_{2 i}\right)$, where $A_{j} \in \mathcal{K}, 1 \leq j \leq 2 k$, and $A_{1} \supseteq A_{2} \supseteq$ $\cdots \supseteq A_{2 k}$. Then $A$ is bounded Hausdorff reducible to $A^{\prime}=A_{1} \oplus \cdots \oplus A_{2 k}$ via $f$ with $f(x)=\left\langle f_{1}(x), \ldots, f_{2 k}(x)\right\rangle$, where $f_{i}$ is a many-one reduction function from $A_{i}$ to $A^{\prime}$.

If $A$ is bounded Hausdorff reducible to a set $B \in \mathcal{K}$ via $f$ then $A=\bigcup_{i=1}^{k}\left(B_{2 i-1}-B_{2 i}\right)$ where $B_{j}=\left\{x \mid f(x)=\left\langle y_{1}, \ldots, y_{2 k}\right\rangle\right.$ and $\left.y_{j} \in B\right\}$. Since $B_{j} \leq_{m}^{p} B$ this is a Hausdorff representation for $A$ over $\mathcal{K}$.

The next lemma is obtained by combining Theorem 3.2 and the above two lemmas.

Lemma 3.5 If $\mathcal{K}$ is a set ring which contains $\mathrm{P}$ and is closed under many-one reductions then $B C(\mathcal{K})=R_{b h d}^{p}(\mathcal{K})=R_{b}^{p}(\mathcal{K})$.

\section{Theorem 3.6}

$$
\begin{aligned}
& \text { 1. } R_{b h d}^{p}\left(R_{c}^{p}(\mathrm{SPARSE})\right)=R_{b}^{p}\left(R_{c}^{p}(\mathrm{SPARSE})\right) \\
& \text { 2. } R_{b h d}^{p}\left(R_{m}^{c o-n p}(\mathrm{SPARSE})\right)=R_{b}^{p}\left(R_{m}^{c c^{-n p}}(\mathrm{SPARSE})\right)
\end{aligned}
$$

Proof In order to prove the two parts it suffices to show that $R_{c}^{p}$ (SPARSE) and $R_{m}^{c 0^{-n p}}$ (SPARSE) are set rings. It is easy to see that $R_{c}^{p}$ (TALLY) and $R_{m}^{c c^{-n p}}$ (TALLY) are set rings. Since SPARSE $\subset R_{c}^{p}$ (TALLY) [BLS92] it holds that $R_{c}^{p}$ (SPARSE) $=$ $R_{c}^{p}$ (TALLY) and $R_{m}^{c c^{-n p}}$ (SPARSE) $=R_{m}^{c c^{-n p}}$ (TALLY) and therefore $R_{c}^{p}$ (SPARSE) and $R_{m}^{c o-n p}(\mathrm{SPARSE})$ are set rings.

A truth-table reduction is honest if there exists a polynomial $p$ such that for every query $y$ on input $x,|x| \leq p(|y|)$. As far as reductions to sparse or tally sets are concerned we can assume that they are honest.

Lemma 3.7 If A reduces to a sparse (tally) set $S$ via a truth-table reduction of a certain type then $A$ reduces honestly to a sparse (respectively, tally) set $S^{\prime}$ via a truth-table reduction of the same type.

Proof Define $S^{\prime}=\left\{\left\langle 0^{n}, y\right\rangle \mid n \geq 0, y \in S\right\}$ and in the new reduction to $S^{\prime}$ replace each query $y$ on input $x$ by the query $\left\langle 0^{|x|}, y\right\rangle$.

In the case of reductions to sparse sets we can even assume that the query length depends only on the input length (via some strictly increasing polynomial).

Lemma 3.8 If $A$ reduces to a sparse set $S$ via a truth-table reduction of a certain type with query generator $g$ then $A$ reduces to a sparse set $S^{\prime}$ via a truth-table reduction of the same type with a query generator $g^{\prime}$ such that for all $x$ the queries generated by $g^{\prime}(x)$ have length $r(|x|)$ for a strictly increasing polynomial $r$. 
Proof Replace each query $y$ by $y 10^{t(|x|)-|y|}$ and $S$ by the sparse set $\left\{z 10^{i} \mid z \in S, i \geq 0\right\}$ where $t$ is a polynomial bounding the computation time of the query generator $g$.

For a class $\mathcal{C}$ of sets and a class $\mathcal{F}$ of functions from $\Sigma^{*}$ to $\Sigma^{*}$ let $\mathcal{C} / \mathcal{F}$ [KL80] be the class of sets $A$ such that there is a set $B \in \mathcal{C}$ and a function $h \in \mathcal{F}$ such that for all $x \in \Sigma^{*}$,

$$
\chi_{A}(x)=\chi_{B}\left(x, h\left(0^{|x|}\right)\right)
$$

Although Karp and Lipton introduced the notion of advice functions in order to characterize nonuniform complexity classes by imposing a quantitative length restriction on the functions in $\mathcal{F}$, we will consider here complexity restricted advice function classes. We refer the reader to [KT90] for a general study of complexity restricted advice functions.

For a set $A$ the class $\Theta(A)$ [LS91] contains all languages $L(M, A)$ accepted by a deterministic polymomial time bounded oracle machine $M$ asking on inputs of length $n$ at most $O(\log n)$ queries to $A$. A deterministic polynomial-time oracle machine $M$ as in the definition above is called a $\Theta$ machine. The $\Theta$ levels of the (relativized) polynomial time hierarchy are defined as $\Theta_{k}^{p}(A)=\Theta\left(\Sigma_{k-1}^{p}(A)\right), k \geq 1$ [Wag90, LS91].

Similarly, the class $\mathrm{F} \Theta(A)$ contains all functions computable by some deterministic polymomial time bounded oracle transducer $M$ asking on inputs of length $n$ at most $O(\log n)$ queries to oracle $A$, and $\mathrm{F}_{k}^{p}(A)=\mathrm{F} \Theta\left(\sum_{k-1}^{p}(A)\right), k \geq 1$.

For further definitions used in this paper we refer the reader to standard books on structural complexity theory (for example, [BDG, Sch86]).

\section{Upper bounds for sparse and tally descriptions}

In this section we consider the following question: If a set $A$ reduces to a sparse set $S$ via a reduction of a certain type, does it follow that $A$ reduces via a reduction of the same type to some sparse set $S^{\prime}$ that is "simple" relative to $A$ ?

We notice that a simplicity argument was already used in the proof of Mahaney's theorem [Mah82] that if NP has sparse hard sets then $\mathrm{P}=$ NP. Mahaney first showed that $\mathrm{P}=\mathrm{NP}$ under the stronger assumption that NP has a sparse complete set. From that the theorem is derived by observing that if a set $A \in \mathrm{NP}$ many-one reduces in polynomial time to a sparse set then it actually many-one reduces to a sparse set in NP. This observation can be formalized as a simplicity result for sets in $R_{m}^{p}$ (SPARSE).

Theorem 4.1 [LS91] If $A \in R_{m}^{p}$ (SPARSE) then there exists a sparse set $S \in R_{m}^{n p}(A)$ such that $A \leq_{m}^{p} S$.

It is easy to see that the same upper bound holds for $R_{c}^{p}$ (SPARSE), i.e., every set $A \in R_{c}^{p}$ (SPARSE) has a sparse $c$-description in $R_{m}^{n p}(A)$. On the other hand, for a set $A$ in $R_{b}^{p}$ (SPARSE) the only known upper bound for the complexity of sparse $b$-descriptions is $\Delta_{3}^{p}(A)$ (this can be seen by a minor modification of the proof that every set $A$ in $\mathrm{P} /$ poly has an advice function computable in $\Delta_{3}^{p}(A)$ [Sch86]).

Surprisingly, it turns out (see the following theorem) that each set $A$ in the reduction class $R_{h d}^{p}\left(R_{c}^{p}\right.$ (SPARSE)), which is larger than $R_{b}^{p}$ (SPARSE), has a sparse $h d(c)$ description in NP $(A)$. From this it follows (see Section 5$)$ that $R_{h d}^{p}\left(R_{c}^{p}(\mathrm{SPARSE})\right.$ ), and therefore $R_{b}^{p}$ (SPARSE) are in $E L_{3}^{\Theta}$. 
Theorem 4.2 For every set $A \in R_{h d}^{p}\left(R_{c}^{p}\right.$ (SPARSE)) there is a sparse set $\hat{S} \in \operatorname{NP}(A)$ such that $A \in R_{h d}^{p}\left(R_{c}^{p}(\hat{S})\right)$.

Proof Let $A$ be a set in $R_{h d}^{p}\left(R_{c}^{p}(S)\right)$ for a sparse set $S$. Composing the Hausdorff and conjunctive reduction functions, we obtain FP functions $g$ and $k$ such that for all $x$,

1. for all $i, 1 \leq i \leq 2 k(x)-1, g(i+1, x) \subseteq S$ implies $g(i, x) \subseteq S$

2. $x \in A \Leftrightarrow$ for some even $i, 2 \leq i \leq 2 k(x), g(i-1, x) \subseteq S$ and $g(i, x) \not \subseteq S$

By Lemma 3.8 we can assume that there exists a strictly increasing polynomial $r$ such that $|y|=r(|x|)$ for all $x$ and all $y \in \bigcup_{i=1}^{2 k(x)} g(i, x)$. In order to prove the theorem we give an $\operatorname{NP}(A)$ algorithm that accepts a sparse set $\hat{S} \subseteq S$ such that $A$ reduces to $\hat{S}$ via $g$ and $k$. In other words, we will show that $\hat{S}$ fulfils the two conditions above for all $x \in \Sigma^{*}$. Intuitively, on input $y$ of length $r(n)$ starting with $T=\emptyset$ the algorithm expands $T$ inside $S^{=r(n)}$ by guessing strings in $\Sigma^{n}$ witnessing that $T$ does not yet fulfil the two conditions above. Finally $y$ is accepted if $y \in T$. The $\operatorname{NP}(A)$ algorithm for $\hat{S}$ is given by the following code ( $p$ is a polynomial bounding the census of the sparse set $S$ ).

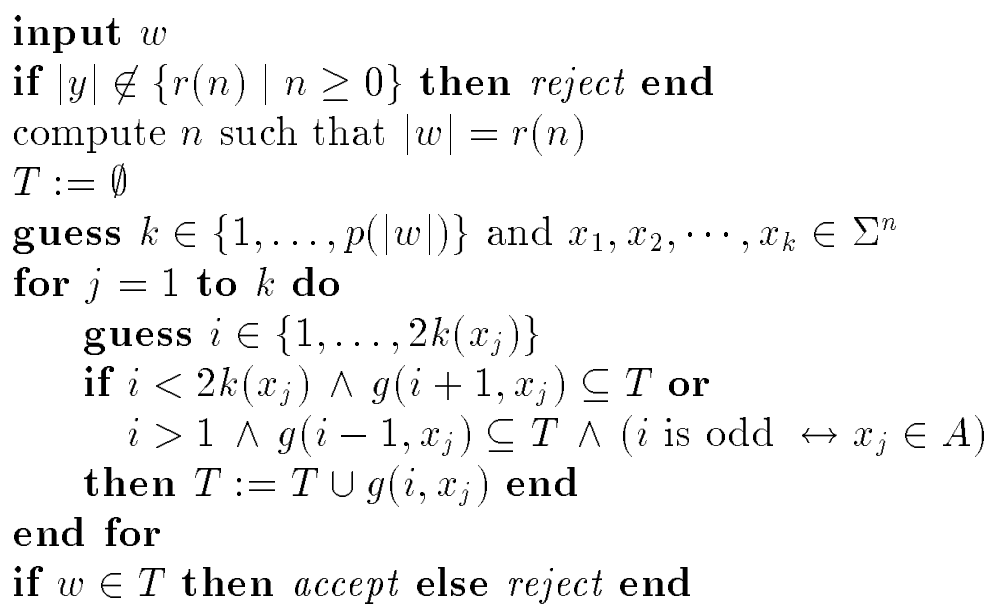

Clearly, the running time of the algorithm is polynomially bounded. We prove that the set $\hat{S}$ accepted by the above algorithm has the required properties by establishing the following claims.

Claim $1 T$ is a subset of $S$ during each step of the computation.

Proof of Claim 1: The claim certainly holds when the computation starts. Therefore it suffices to show that whenever a set $g\left(i, x_{j}\right)$ is included in $T$ then it holds that $g\left(i, x_{j}\right) \subseteq S$ provided that $T \subseteq S$. But this immediately follows from the fact that $T$ is only expanded by $g\left(i, x_{j}\right)$ if

$$
i<2 k\left(x_{j}\right) \wedge g\left(i+1, x_{j}\right) \subseteq T
$$

or

$$
i>1 \wedge g\left(i-1, x_{j}\right) \subseteq T \wedge\left(i \text { is odd } \leftrightarrow x_{j} \in A\right)
$$


Claim 2 For every input of length $r(n)$ there is a computation path along which the set $T=\hat{S}^{=r(n)}$ is computed, and $\hat{S}^{=r(n)}$ fulfils conditions 1 and 2 for all $x$ of length $n$.

Proof of Claim 2: Let $T(n)$ be a set of maximum cardinality that is computed along some path $\tau$ on some input of length $r(n)$. Since $T(n) \subseteq S^{=r(n)}$ by Claim 1 it follows that $|T(n)| \leq p(r(n))$. We first show that $T(n)=\hat{S}^{=r(n)}$. In order to derive a contradiction assume that there is an input $y \in \hat{S}^{=r(n)}$ that is not contained in $T(n)$. Then clearly $|T(n)|<p(r(n))$ holds by Claim 1 since otherwise $T(n)=S^{\leq r(n)}$. Therefore we can assume w.l.o.g. that the value of $k$ guessed on path $\tau$ is smaller than $p(r(n))$ since we can remove all strings $x_{j}$ from the list that don't expand $T$ along $\tau$.

Consider now some computation path accepting $y$. Since $y \notin T(n)$ the set $T$ computed along this path is not contained in $T(n)$. Let $T^{\prime}$ be the value of $T$ just before an element $q$ not in $T(n)$ is included to $T$ for the first time, and let $j_{0}$ and $i_{0}$ be the corresponding values of $j$ and $i$, respectively, i.e., $q \in g\left(i_{0}, x_{j_{0}}\right)$. Then it holds that $T^{\prime} \subseteq T(n)$. Thus, adding $x_{j_{0}}$ to the list of strings guessed along path $\tau$ and guessing $i_{0}$ in the $j_{0}$ th iteration of the for-loop gives a computation path $\tau^{\prime}$ that computes the set $T(n) \cup g\left(i_{0}, x_{j_{0}}\right)$, contradicting the maximality of $|T(n)|$.

Using the maximality of $|T(n)|$ it is easy to see that $T(n)=\hat{S}^{=r(n)}$ fulfils conditions 1 and 2 for all $x$ of length $n$ since otherwise $T(n)$ could be expanded.

Because the query sets for inputs of different lengths are disjoint conditions 1 and 2 are fulfilled by $\hat{S}$ for all inputs $x$, i.e., $A$ reduces to $\hat{S}$ via the given reduction.

Since the given $h d(c)$-reduction function is not modified in the proof of Theorem 4.2 we immediately obtain the following corollary.

Corollary 4.3 For every set $A \in R_{b h d}^{p}\left(R_{c}^{p}(\mathrm{SPARSE})\right)$ there is a sparse set $S^{\prime} \in \mathrm{NP}(A)$ such that $A \in R_{b h d}^{p}\left(R_{c}^{p}\left(S^{\prime}\right)\right)$.

Since $R_{b h d}^{p}\left(R_{c}^{p}(\mathrm{SPARSE})\right)=R_{b}^{p}\left(R_{c}^{p}(\mathrm{SPARSE})\right)$ by Theorem 3.6 and since every bounded Hausdorff reduction is also a bounded truth-table reduction we additionally have the following simplicity result for $R_{b}^{p}\left(R_{c}^{p}\right.$ (SPARSE)).

Corollary 4.4 For every set $A \in R_{b}^{p}\left(R_{c}^{p}\right.$ (SPARSE)) there is a sparse set $S^{\prime} \in \operatorname{NP}(A)$ such that $A \in R_{b}^{p}\left(R_{c}^{p}\left(S^{\prime}\right)\right)$.

Next we consider sets in $R_{d}^{p}$ (SPARSE) and show that every set $A$ in $R_{d}^{p}$ (SPARSE) has a sparse $d$-description in $\mathrm{NP}(A \oplus \mathrm{SAT}) / \mathrm{F}_{2}^{p}(A)$. Furthermore we show that there exists a set in $\mathrm{NP}(A) / \mathrm{F}_{2}^{p}(A)$ such that every element $\left\langle 0^{n}, W\right\rangle$ in this set encodes a finite set $W$ to which $A$ is disjunctively reducible with respect to strings of length $n$, i.e., $A^{=n} \leq_{d}^{p} W$ via the given disjunctive reduction function (cf. the notion of $\operatorname{CIR}(A)$ $[\mathrm{KoSc85}])$. Our proof technique is a refinement and an extension of the one developed in $\left[\mathrm{AHH}^{+} 92\right]$ where it is shown that for every set $A$ in $R_{d}^{p}$ (SPARSE) there is a sparse set $S^{\prime} \in \mathrm{P}(\mathrm{NP}(A))$ such that $A \in R_{d}^{p}\left(S^{\prime}\right)$.

Theorem 4.5 Let $A$ be a set in $R_{d}^{p}$ (SPARSE) witnessed by the sparse set $S$ and the reduction function $f$. Then

1. there exists a set $C \subseteq\left\{\left\langle 0^{n}, W\right\rangle \mid A \leq_{d}^{p} W\right.$ via $f$ for inputs of length $\left.n\right\}$ and a polynomial $p$ such that $C \in \mathrm{NP}(A) / \mathrm{F}_{2}^{p}(A)$ and for every $n$ there is at least one pair of the form $\left\langle 0^{n}, W\right\rangle$ in $C \leq p(n)$, 
2. there exists a sparse set $\hat{S} \in \mathrm{NP}(A \oplus \mathrm{SAT}) / \mathrm{F}_{2}^{p}(A)$ such that $A \leq_{d}^{p} \hat{S}$ via $f$.

Proof By Lemma 3.8 we can assume that $A$ reduces to a sparse set via a reduction function $f$ such that for all $x$ and $y \in f(x),|y|=r(|x|)$ and $|f(x)| \leq r(|x|)$, where $r$ is a strictly increasing polynomial. Then it holds for all $x$ that $x \in A$ if and only if $f(x) \cap S^{=r(|x|)} \neq \emptyset$. We construct sparse sets $S_{1} \subseteq A$ and $S_{2} \subseteq \bar{A}$ which define a sparse set $\hat{S}=\bigcup_{y \in S_{1}} f(y)-\bigcup_{y \in S_{2}} f(y)$ such that for all $x, x \in A$ if and only if $f(x) \cap \hat{S} \neq \emptyset$. Consider the set $L$ defined by

$$
\begin{aligned}
& \left\langle 0^{n},\left\langle x_{1}, \ldots, x_{j}\right\rangle,\left\langle y_{1}, \ldots, y_{k}\right\rangle\right\rangle \in L \Leftrightarrow \\
& \quad \text { for all } l, 1 \leq l \leq j, x_{l} \in A^{=n} \wedge f\left(x_{l}\right) \cap\left(\bigcup_{1 \leq i<l} f\left(x_{i}\right)-\bigcup_{i=1}^{k} f\left(y_{i}\right)\right)=\emptyset \\
& \quad \text { and } \\
& \quad \text { for all } l, 1 \leq l \leq k, y_{l} \in \bar{A}^{=n} \wedge f\left(y_{l}\right) \cap\left(\bigcup_{i=1}^{j} f\left(x_{i}\right)-\bigcup_{1 \leq i<l} f\left(y_{i}\right)\right) \neq \emptyset
\end{aligned}
$$

Clearly $L$ is in $\mathrm{P}(A)$. Note that for $\left\langle 0^{n},\left\langle x_{1}, \ldots, x_{j}\right\rangle,\left\langle y_{1}, \ldots, y_{k}\right\rangle\right\rangle$ in $L$ the set $\bigcup_{i=1}^{k} f\left(y_{i}\right)$ is a subset of $\bar{S}$ and therefore it holds for every $l, 1 \leq l \leq k$, that $f\left(x_{l}\right) \cap \bigcup_{1 \leq i<l} f\left(x_{i}\right) \cap S=\emptyset$, i.e., $f\left(x_{l}\right)$ generates at least one query in $S$ that is not covered by any of the sets $f\left(x_{1}\right), \ldots, f\left(x_{l-1}\right)$. The advice that we use are the values of the following two functions,

$$
\begin{gathered}
j(n)=\max \left\{j \mid \exists k \exists x_{1}, \ldots, x_{j}, y_{1}, \ldots, y_{k}:\left\langle 0^{n},\left\langle x_{1}, \ldots, x_{j}\right\rangle,\left\langle y_{1}, \ldots, y_{k}\right\rangle\right\rangle \in L\right\} \\
k(n)=\max \left\{k \mid \exists x_{1}, \ldots, x_{j(n)}, y_{1}, \ldots, y_{k}:\left\langle 0^{n},\left\langle x_{1}, \ldots, x_{j(n)}\right\rangle,\left\langle y_{1}, \ldots, y_{k}\right\rangle\right\rangle \in L\right\}
\end{gathered}
$$

Claim 3 For all $n$ it holds that $j(n) \leq$ census $_{S}\left(1^{r(n)}\right)$ and $k(n) \leq r(n) \cdot$ census $_{S}\left(1^{r(n)}\right)$.

Proof of Claim 3: Let $\left\langle 0^{n},\left\langle x_{1}, \ldots, x_{j(n)}\right\rangle,\left\langle y_{1}, \ldots, y_{k}\right\rangle\right\rangle \in L$. As noted above, it holds for every $l, 1 \leq l \leq j(n)$, that $f\left(x_{l}\right) \cap \bigcup_{1 \leq i<l} f\left(x_{i}\right) \cap S=\emptyset$. Since $f\left(x_{i}\right) \cap S \neq \emptyset$, $1 \leq i \leq j(n)$, it follows that

$$
\operatorname{census}_{S}\left(1^{r(n)}\right) \geq\left|\bigcup_{1 \leq i \leq j(n)} f\left(x_{i}\right) \cap S\right|=\sum_{i=1}^{j(n)}\left|f\left(x_{i}\right) \cap S\right| \geq j(n)
$$

This proves the first part of the claim. The second part follows immediately by the definition of $L$ since for all $x,|f(x)| \leq r(|x|)$, i.e., $\left|\bigcup_{1 \leq i \leq j(n)} f\left(x_{i}\right)\right| \leq j(n) \cdot r(n)$.

Since $r(n)$ and $\operatorname{census}_{S}\left(1^{n}\right)$ are bounded by some polynomial the functions $j(n)$ and $k(n)$ are also polynomially bounded, and the following claim can be easily proved.

Claim 4 On input $0^{n}, j(n)$ and $k(n)$ can be computed by an $\mathrm{F}_{2}^{p}(A)$ computation.

Every element $z=\left\langle 0^{n},\left\langle x_{1}, \ldots, x_{j(n)}\right\rangle,\left\langle y_{1}, \ldots, y_{k(n)}\right\rangle\right\rangle$ in $L$ contains enough information to construct a set $S_{z}$ containing at most $r(n) \cdot j(n)$ elements such that $A^{=n} \leq_{d}^{p} S_{z}$ via $f$.

Claim 5 Let $\left\langle 0^{n},\left\langle x_{1}, \ldots, x_{j(n)}\right\rangle,\left\langle y_{1}, \ldots, y_{k(n)}\right\rangle\right\rangle \in L$. Then for all $x \in \Sigma^{n}$ it holds that $x \in A$ if and only if $f(x) \cap\left(\bigcup_{i=1}^{j(n)} f\left(x_{i}\right)-\bigcup_{i=1}^{k(n)} f\left(y_{i}\right)\right) \neq \emptyset$. 
Proof of Claim 5: In order to derive a contradiction let $x \in \Sigma^{n}$ and assume that $x \in A$ but $f(x) \cap\left(\bigcup_{i=1}^{j(n)} f\left(x_{i}\right)-\bigcup_{i=1}^{k(n)} f\left(y_{i}\right)\right)=\emptyset$. Then it follows that the string $\left\langle 0^{n},\left\langle x_{1}, \ldots, x_{j(n)}, x\right\rangle,\left\langle y_{1}, \ldots, y_{k(n)}\right\rangle\right\rangle$ is contained in $L$ which contradicts the maximality of $j(n)$. To show the converse assume that $x \notin A$ and $f(x) \cap\left(\bigcup_{i=1}^{j(n)} f\left(x_{i}\right)-\bigcup_{i=1}^{k(n)} f\left(y_{i}\right)\right) \neq \emptyset$. Then $\left\langle 0^{n},\left\langle x_{1}, \ldots, x_{j(n)}\right\rangle,\left\langle y_{1}, \ldots, y_{k(n)}, x\right\rangle\right\rangle \in L$ contradicting the maximality of $k(n)$.

Now consider the sets $C$ and $C^{\prime}$ defined by

$$
\left\langle 0^{n}, W, j, k\right\rangle \in C^{\prime} \Leftrightarrow \exists\left\langle 0^{n},\left\langle x_{1}, \ldots, x_{j}\right\rangle,\left\langle y_{1}, \ldots, y_{k}\right\rangle\right\rangle \in L: W=\bigcup_{i=1}^{j} f\left(x_{i}\right)-\bigcup_{i=1}^{k} f\left(y_{i}\right)
$$

and

$$
\left\langle 0^{n}, W\right\rangle \in C \Leftrightarrow\left\langle 0^{n}, W, j(n), k(n)\right\rangle \in C^{\prime}
$$

Clearly, $C^{\prime} \in \mathrm{NP}(A)$, and therefore $C \in \mathrm{NP}(A) / \mathrm{F \Theta}_{2}^{p}(A)$. By Claim 5 it holds for every $\left\langle 0^{n}, W\right\rangle \in C$ that $A^{=n} \leq_{d}^{p} W$ via $f$. Furthermore, by the definitions of $L$ and $C$ it follows for all $\left\langle 0^{n}, W\right\rangle \in C$ that $W \subseteq \Sigma^{r(n)}$ and $|W| \leq r(n) \cdot j(n)$. This proves the first part of the theorem.

In order to prove the second part we select for every $n$ the lexicographically smallest pair $\left\langle 0^{n}, W_{n}\right\rangle$ in $C$ and define the desired sparse set $\hat{S}$ as the union of all the $W_{n}, n \geq 0$. Consider the NP set $D$ defined by

$\left\langle 0^{n}, W, j, k\right\rangle \in D \Leftrightarrow$ there exists a string $\left\langle 0^{n},\left\langle x_{1}, \ldots, x_{j}\right\rangle,\left\langle y_{1}, \ldots, y_{k}\right\rangle\right\rangle$ such that for all $l, 1 \leq l \leq j, f\left(x_{l}\right) \cap W \neq \emptyset \wedge f\left(x_{l}\right) \cap\left(\bigcup_{1 \leq i<l} f\left(x_{i}\right)-\bigcup_{i=1}^{k} f\left(y_{i}\right)\right)=\emptyset$ and for all $l, 1 \leq l \leq k, f\left(y_{l}\right) \cap W=\emptyset \wedge f\left(y_{l}\right) \cap\left(\bigcup_{i=1}^{j} f\left(x_{i}\right)-\bigcup_{1 \leq i<l} f\left(y_{i}\right)\right) \neq \emptyset$ and $\left\langle 0^{n}, \bigcup_{i=1}^{j} f\left(x_{i}\right)-\bigcup_{i=1}^{k} f\left(y_{i}\right)\right\rangle<\left\langle 0^{n}, W\right\rangle$

Claim 6 A string $\left\langle 0^{n}, W, j(n), k(n)\right\rangle \in C^{\prime}$ is contained in $D$ if and only if there exists a string $\left\langle 0^{n}, W^{\prime}\right\rangle \in C$ which is lexicographically smaller than $\left\langle 0^{n}, W\right\rangle$.

Proof of Claim 6: Let $\left\langle 0^{n}, W, j(n), k(n)\right\rangle$ be a string in $C^{\prime}$. Then there exists a string $\left\langle 0^{n},\left\langle x_{1}, \ldots, x_{j(n)}\right\rangle,\left\langle y_{1}, \ldots, y_{k(n)}\right\rangle\right\rangle \in L$ such that $W=\bigcup_{i=1}^{j(n)} f\left(x_{i}\right)-\bigcup_{i=1}^{k(n)} f\left(y_{i}\right)$. Thus it follows by Claim 5 that for all $x$ of length $n, x \in A$ if and only if $f(x) \cap W \neq \emptyset$, and the claim follows by the definition of $D$.

An immediate consequence of Claim 6 is

Claim 7 For every $n$ there is exactly one element of the form $\left\langle 0^{n}, W\right\rangle$ in $C^{\prime}-D$.

Now we are ready to define the sparse set $\hat{S}=\bigcup_{n}\left\{W \mid\left\langle 0^{n}, W\right\rangle \in C-D\right\}$. For $r(n)=|w|$ the following algorithm accepts an input $\langle w, j(n), k(n)\rangle$ if and only if $w$ is in $\hat{S}$.

input $\langle w, j, k\rangle$

guess $n \leq|w|$

if $|w| \neq r(n)$ then reject end

guess $x_{1}, \ldots, x_{j}, y_{1}, \ldots, y_{k} \in \Sigma^{n}$

if $\left\langle 0^{n},\left\langle x_{1}, \ldots, x_{j}\right\rangle,\left\langle y_{1}, \ldots, y_{k}\right\rangle\right\rangle \notin L$ then reject end

$W:=\bigcup_{i=1}^{j} f\left(x_{i}\right)-\bigcup_{i=1}^{k} f\left(y_{i}\right)$

if $\left\langle 0^{n}, W, j, k\right\rangle \notin D$ and $w \in W$ then accept else reject end 
Since the first if-statement queries oracle $L$ (which is in $\mathrm{P}(A)$ ), the second if-statement queries oracle $D$ (which is in NP) and since the advice (namely $j(n)$ and $k(n)$ ) can be computed in $\mathrm{F}_{2}^{p}(A)$ it follows that $\hat{S} \in \mathrm{NP}(A \oplus \operatorname{SAT}) / \mathrm{F}_{2}^{p}(A)$.

The following theorem shows in general that a simplicity result for a reduction class $R_{r}^{p}$ (SPARSE) can be translated into a simplicity result for the reduction class $R_{1-t t}^{p}\left(R_{r}^{p}(\mathrm{SPARSE})\right)$.

Theorem 4.6 Let $\mathcal{C}$ be a relativizable complexity class closed under join such that $\mathcal{C}(A \oplus B)=\mathcal{C}(A)=\mathcal{C}(\bar{A})$ for all sets $A \subseteq \Sigma^{*}$ and $B \in \mathrm{P}$. If every set $L$ in $R_{r}^{p}$ (SPARSE) has a sparse $r$-description in $\mathcal{C}(L)$ then every set $A$ in $R_{1-t t}^{p}\left(R_{r}^{p}\right.$ (SPARSE)) has a sparse 1-tt $(r)$-description in $\mathcal{C}(A)$.

Proof Let $A$ be in $R_{1-t t}^{p}\left(R_{r}^{p}\right.$ (SPARSE)) witnessed by a generator $f$ and a set $B \in R_{r}^{p}(S)$ for some sparse set $S$. Then it holds for all $x$,

$$
x \in A \Leftrightarrow[f(x)=\langle q, i d\rangle \text { and } q \in B] \text { or }[f(x)=\langle q, n e g\rangle \text { and } q \notin B]
$$

Let $X=\{x \mid f(x)=\langle q, i d\rangle$ for some $q\}$ and $Y=\{x \mid f(x)=\langle q, n e g\rangle$ for some $q\}$. Then it is clear that $\{X, Y\}$ is a partition of $\Sigma^{*}$. Furthermore, $A \cap X \leq_{r}^{p} S$ and $\overline{A \cap Y} \leq_{r}^{p} S$. Now, using the assumption that every set $L$ in the class $R_{r}^{p}$ (SPARSE) has a sparse $r$-description in $\mathcal{C}(L)$ it follows that there are sparse sets $S_{1} \in \mathcal{C}(A \cap X)$ and $S_{2} \in \mathcal{C}(\overline{A \cap Y})$ such that

$$
A \cap X \leq_{r}^{p} S_{1} \text { and } \overline{A \cap Y} \leq_{r}^{p} S_{2}
$$

via reduction functions $g_{1}$ and $g_{2}$, respectively. Now the proof is completed by combining the two $r$-reductions $g_{1}$ and $g_{2}$ to a reduction witnessing $A \in R_{1-t t}^{p}\left(R_{r}^{p}\left(S_{1} \oplus S_{2}\right)\right)$ and by observing that $S_{1} \oplus S_{2}$ is a sparse set in $\mathcal{C}(A)$.

Corollary 4.7 For every set $A$ in $R_{1-t t}^{p}\left(R_{d}^{p}\right.$ (SPARSE)) there is a sparse set $S^{\prime}$ in $\mathrm{NP}(A \oplus \mathrm{SAT}) / \mathrm{F}_{2}^{p}(A)$ such that $A \in R_{1-t t}^{p}\left(R_{d}^{p}\left(S^{\prime}\right)\right)$.

Next we consider nondeterministic reduction classes to sparse sets. The notion of $\leq_{m}^{c c^{-n p}}$ reducibility can be seen as a generalization of the deterministic polynomial-time conjunctive reducibility.

Definition 4.8 A set $A$ is co-np many-one reducible to a set $B$ (denoted $A \leq_{m}^{c o-n p} B$ ) if there exists a polynomial-time nondeterministic Turing transducer $M$ such that for every $x \in \Sigma^{*}, x \in A$ if and only if all outputs of $M$ on input $x$ are in $B$.

Note that $A \leq_{m}^{c^{-n p}} B$ if and only if $\bar{A} \leq_{m}^{n p} \bar{B}$ where $\leq_{m}^{n p}$ is the more familiar polynomialtime nondeterministic many-one reducibility [LLS75]. Clearly, for every set $B, R_{c}^{p}(B)$ is contained in $R_{m}^{c o-n p}(B)$, and $R_{m}^{c o-n p}(B)$ is closed downward under $\leq_{c}^{p}$ and $\leq_{m}^{c o-n p}$ reductions.

Theorem 4.9 For every set $A \in R_{m}^{c o-n p}$ (SPARSE) there exists a sparse set $S^{\prime} \in R_{m}^{n p}(A)$ such that $A \in R_{m}^{c o-n p}\left(S^{\prime}\right)$. 
Proof Let $S$ be a sparse set and let $M$ be an NP transducer witnessing $A \in R_{m}^{c o-n p}(S)$. W.1.o.g. we can assume that there is a polynomial $p$ such that for all output strings $y$ of $M$ on input $x$ it holds that $|x| \leq p(|y|)$. Consider the following subset $S^{\prime}$ of $S$.

$$
S^{\prime}=\{y \mid \exists x \in A: M \text { on input } x \text { outputs } y\}
$$

Then $A \in R_{m}^{c o-n p}\left(S^{\prime}\right)$ via $M$. Furthermore, the following NP transducer witnesses $S^{\prime} \leq_{m}^{n p} A$.

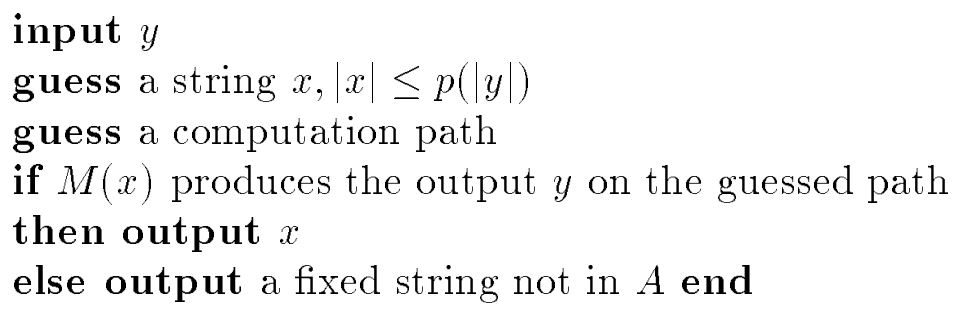

The next theorem shows how to construct (relative to $A$ ) small descriptions for sets in the reduction class $R_{h d}^{p}\left(R_{m}^{c o-n p}(\mathrm{SPARSE})\right)$.

Theorem 4.10 For every set $A \in R_{h d}^{p}\left(R_{m}^{c o-n p}(\mathrm{SPARSE})\right)$ there is a sparse set $S^{\prime} \in$ $\mathrm{NP}(\mathrm{SAT} \oplus A)$ such that $A \in R_{h d}^{p}\left(R_{m}^{c o-n p}\left(S^{\prime}\right)\right)$.

Proof The proof is quite similar to that of Theorem 4.2 and therefore we omit most of the details. Let $A$ be a set in $R_{h d}^{p}\left(R_{m}^{c o-n p}(S)\right)$ for a sparse set $S$. Again we can combine the Hausdorff reduction function and the NP transducer witnessing the co-np many-one reduction to obtain an $\mathrm{FP}$ function $k$ and an NP transducer $M$ such that the following conditions are fulfilled for all $x$.

1. for all $i, 1 \leq i \leq 2 k(x)-1$, if all outputs of $M(i+1, x)$ are contained in $S$ then also all outputs of $M(i, x)$ are contained in $S$

2. $x \in A \Leftrightarrow$ for some even $i, 2 \leq i \leq 2 k(x)$, all outputs of $M(i-1, x)$ are contained in $S$ but some output of $M(i, x)$ is not contained in $S$

We can assume that there exists a strictly increasing polynomial $r$ such that $|y|=r(n)$ for all outputs $y$ of $M$ on any input $(i, x)$ such that $1 \leq i \leq 2 k(x)$ and $|x|=n$.

We give an $\operatorname{NP}(\mathrm{SAT} \oplus A)$ algorithm that accepts a sparse set $\hat{S} \subseteq S$ such that $A$ reduces to $\hat{S}$ via $M$ and $k$.

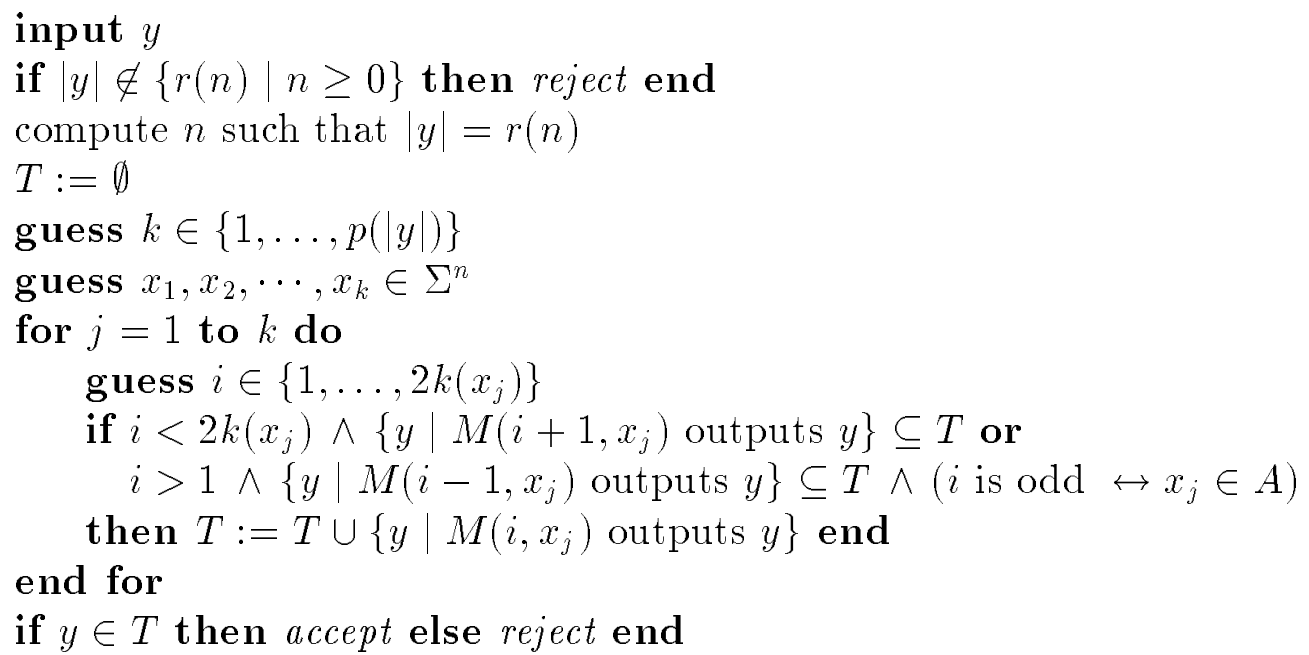


The reason why we need the additional SAT oracle is to test for the inclusions $\{y \mid$ $M\left(i+1, x_{j}\right)$ outputs $\left.y\right\} \subseteq T$ and $\left\{y \mid M\left(i-1, x_{j}\right)\right.$ outputs $\left.y\right\} \subseteq T$, and to compute the set $\left\{y \mid M\left(i, x_{j}\right)\right.$ outputs $\left.y\right\}$. The proof is completed by the following claims which can be proved analogously to Claims 1 and 2 of the proof of Theorem 4.2.

Claim $1 T$ is a subset of $S$ during each step of the computation.

Claim 2 For every input of length $r(n)$ there is a computation path along which the set $T=\hat{S}^{=r(n)}$ is computed, and $\hat{S}^{-r(n)}$ fulfils conditions 1 and 2 for all $x$ of length $n$.

The following corollary is an immediate consequence of Theorems 4.10 and 3.6.

Corollary 4.11 For every set $A \in R_{b}^{p}\left(R_{m}^{c o-n p}(\mathrm{SPARSE})\right)$ there is a sparse set $S^{\prime} \in$ $\operatorname{NP}(\operatorname{SAT} \oplus A)$ such that $A \in R_{b}^{p}\left(R_{m}^{c o-n p}\left(S^{\prime}\right)\right)$.

At the end of this section we consider reduction classes to tally sets. The class $R_{c}^{p}$ (TALLY) $\cap R_{d}^{p}$ (TALLY) is of particular interest since it coincides with the class IC[log,poly] of sets containing only strings of low instance complexity $\left[\mathrm{AHH}^{+} 92\right]$. IC [log,poly] and the notion of instance complexity were introduced by Ko, Orponen, Schöning, and Watanabe (see [OKSW]). A set $A$ is in IC[log,poly] if there exist a constant $c>0$, a polynomial $t$ and a set $\Pi \subseteq \Sigma^{*}$ of programs such that for every $x \in \Sigma^{*}$ the following conditions are fulfilled.

1. there exists a $p \in \Pi \leq c \log (|x|)$ that holds on input $x$ in time $t(|x|)$, and

2. every $p \in \Pi$ that halts on input $x$ decides the membership of $x$ in $A$.

Theorem $4.12\left[\mathbf{A H H}^{+} \mathbf{9 2}\right] \mathrm{IC}[\log$, poly $]=R_{c}^{p}(\mathrm{TALLY}) \cap R_{d}^{p}(\mathrm{TALLY})$.

Let $\Pi$ be the set of programs for $A \in \mathrm{IC}\left[\log\right.$, poly]. Intuitively, $A$ is in $R_{c}^{p}$ (TALLY) $\cap$ $R_{d}^{p}$ (TALLY) because $\Pi$ can be encoded into a tally set $T\left(T=\left\{0^{\text {num }(p)} \mid p \in \Pi\right\}\right.$ where $\operatorname{num}(p)$ is the number whose binary representation is given by $1 p)$. Then $A$ conjunctively reduces to $\bar{T}$ via a reduction function that produces on input $x$ all encodings of small programs which reject $x$ in polynomial time, and $A$ disjunctively reduces to $T$ via a reduction function that generates on input $x$ all encodings of small programs which accept $x$ in polynomial time. Before we state our simplicity result for sets in IC $\left[\log\right.$, poly] we give a direct proof for the containment of $R_{c}^{p}(\mathrm{TALLY}) \cap R_{d}^{p}$ (TALLY) in $\bigcup_{T \in \text { TALLY }} R_{c}^{p}(\bar{T}) \cap R_{d}^{p}(T)$.

Lemma 4.13 For every set $A \in R_{c}^{p}$ (TALLY) $\cap R_{d}^{p}$ (TALLY) there is a tally set $T$ such that $A \leq_{c}^{p} \bar{T}$ and $A \leq_{d}^{p} T$.

Proof Let $T_{1}, T_{2}$ be tally sets and let $f, g$ be reduction functions witnessing $A \in R_{c}^{p}\left(T_{1}\right)$ and $A \in R_{d}^{p}\left(T_{2}\right)$, respectively, i.e. $x \in A \Leftrightarrow f(x) \subseteq T_{1} \Leftrightarrow g(x) \cap T_{2} \neq \emptyset$ for all $x$. Define the tally set $T$ as follows

$$
T=\left\{0^{2 i+1} \mid 0^{i} \notin T_{1}\right\} \cup\left\{0^{2 i} \mid 0^{i} \in T_{2}\right\} .
$$

Furthermore, consider the following reduction functions $f^{\prime}$ and $g^{\prime}$

$$
f^{\prime}(x)=\left\{0^{2 i+1} \mid 0^{i} \in f(x)\right\}, g^{\prime}(x)=\left\{0^{2 i} \mid 0^{i} \in g(x)\right\} .
$$

Then $A$ reduces conjunctively to $\bar{T}$ via $f^{\prime}$ and disjunctively to $T$ via $g^{\prime}$. 
Theorem 4.14 For every set $A \in R_{c}^{p}(\mathrm{TALLY}) \cap R_{d}^{p}$ (TALLY) there is a tally set $T^{\prime} \in$ $\mathrm{P}(\mathrm{SAT} \oplus A)$ such that $A \in R_{c}^{p}\left(T^{\prime}\right) \cap R_{d}^{p}\left(T^{\prime}\right)$.

Proof By Lemma 4.13 we can assume that for $A \in R_{c}^{p}$ (TALLY) $\cap R_{d}^{p}$ (TALLY) there are honest polynomial time reduction functions $f$ and $g$, and a tally set $T$ such that

$$
x \in A \Leftrightarrow f(x) \cap T=\emptyset \Leftrightarrow g(x) \cap T \neq \emptyset
$$

Let $p$ be a polynomial such that $|y| \leq p(|x|)$ and $|x| \leq p(|y|)$ for all queries $y$ in $f(x) \cup g(x)$. Consider the following procedure for $T^{\prime}$.

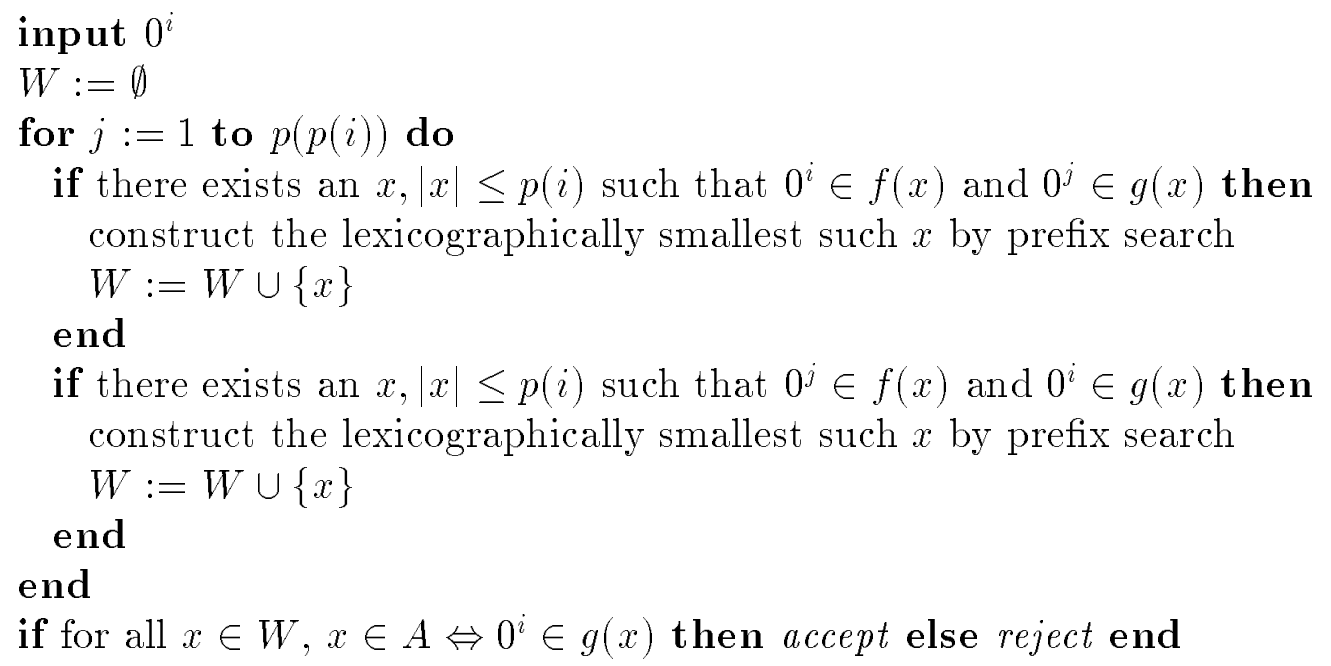

If we interpret $0^{i}$ and $0^{j}$ as encodings of programs $p_{i}$ and $p_{j}$, respectively, (i.e., a program $p_{k}$ accepts an input $x$ if $0^{k} \in g(x)$ and $p_{k}$ rejects $x$ if $\left.0^{k} \in f(x)\right)$ then $0^{i}$ is in $T^{\prime}$ if there is no program $p_{j}$ that decides some input $x$ differently, and $p_{i}$ is wrong on $x$. It is easy to see that the set $W$ can be computed using SAT as oracle and that the acceptance condition can be evaluated asking oracle $A$. In order to show that $T$ can be replaced by $T^{\prime}$ we prove the following claims.

Claim $1 T \subseteq T^{\prime}$

Proof of Claim 1: An input $0^{i}$ is only rejected by the above procedure if either there is a string $x \in A$ such that $0^{i} \in f(x)$ or there is a string $x \notin A$ such that $0^{i} \in g(x)$. In both cases it follows that $0^{i} \notin T$.

Claim 2 For all $x, g(x) \cap T^{\prime} \neq \emptyset \Rightarrow f(x) \cap T=\emptyset$.

Proof of Claim 2: Let $0^{i}$ be in $g(x) \cap T^{\prime}$ and assume that $0^{j} \in f(x)$. Since $0^{i}$ is accepted there exists a string $x^{\prime} \in A$ such that $0^{i} \in g\left(x^{\prime}\right)$ and $0^{j} \in f\left(x^{\prime}\right)$. Since $x^{\prime} \in A$, it follows that $f\left(x^{\prime}\right) \cap T=\emptyset$, and therefore $0^{j} \notin T$.

Claim 3 For all $x, f(x) \cap T^{\prime} \neq \emptyset \Rightarrow g(x) \cap T=\emptyset$.

Proof of Claim 3: Let $0^{i}$ be in $f(x) \cap T^{\prime}$ and assume that $0^{j} \in g(x)$. Since $0^{i}$ is accepted there exists a string $x^{\prime} \notin A$ such that $0^{i} \in f\left(x^{\prime}\right)$ and $0^{j} \in g\left(x^{\prime}\right)$. Since $x^{\prime} \notin A$, 
it follows that $g\left(x^{\prime}\right) \cap T=\emptyset$, and therefore $0^{j} \notin T$. $\square$ Now the proof can be completed by showing that $A \leq_{c}^{p} \overline{T^{\prime}}$ via $f$ and $A \leq_{d}^{p} T^{\prime}$ via $g$.

$$
\begin{aligned}
& x \in A \Rightarrow g(x) \cap T \neq \emptyset \stackrel{(\text { Claim } 1)}{\Rightarrow} g(x) \cap T^{\prime} \neq \emptyset \stackrel{\text { (Claim 2) }}{\Rightarrow} f(x) \cap T=\emptyset \Rightarrow x \in A \\
& x \notin A \Rightarrow f(x) \cap T \neq \emptyset \stackrel{(\text { Claim } 1)}{\Rightarrow} f(x) \cap T^{\prime} \neq \emptyset \stackrel{\text { (Claim 3) }}{\Rightarrow} g(x) \cap T=\emptyset \Rightarrow x \notin A
\end{aligned}
$$

Essentially by the same proof we obtain the following theorem.

Theorem 4.15 For every set $A \in R_{m}^{n p}$ (TALLY) $\cap R_{m}^{c o-n p}$ (TALLY) there is a tally set $T^{\prime} \in \mathrm{P}(\mathrm{SAT} \oplus A)$ such that $A \in R_{m}^{n p}\left(T^{\prime}\right) \cap R_{m}^{c o-n p}\left(T^{\prime}\right)$.

The class IC [log, poly] is known to be closed downward under polynomial time bounded truth-table reductions [OKSW]. It is interesting to note that IC[log, poly] and $R_{m}^{n p}(\mathrm{TALLY}) \cap R_{m}^{c o-n p}$ (TALLY) are also closed downward under polynomial time Hausdorff reductions.

\section{Theorem 4.16}

1. $R_{h d}^{p}(\mathrm{IC}[\log$, poly $])=\mathrm{IC}[\log$, poly $]$

2. $R_{h d}^{p}\left(R_{m}^{n p}(\mathrm{TALLY}) \cap R_{m}^{c 0^{-n p}}(\mathrm{TALLY})\right)=R_{m}^{n p}(\mathrm{TALLY}) \cap R_{m}^{c o-n p}(\mathrm{TALLY})$

Proof We prove the first part of the theorem. The second part can be proved similarly. Let $A \leq_{h d}^{p} B$ for a set $B \in \mathrm{IC}[\log$, poly] via FP functions $h$ and $k$, i.e., $x \in A$ if and only if there is an $i \in\{1, \ldots, k(x)\}$ such that $h(2 i-1, x) \in B$ but $h(2 i, x) \notin B$. By Lemma 4.13 there is a tally set $T$ such that $B \leq_{c}^{p} \bar{T}$ via $f$ and $B \leq_{d}^{p} T$ via $g$. Consider the tally set $T^{\prime}=\{\langle y, z\rangle \mid y, z \in T\}$ and the reduction function

$$
g^{\prime}(x)=\bigcup_{l=1}^{k(x)}\{\langle y, z\rangle \mid y \in g(h(2 l-1, x)), z \in f(h(2 l, x))\}
$$

If $x \in A$ then there is an $i \in\{1, \ldots, k(x)\}$ such that $h(2 i-1, x) \in B$ but $h(2 i, x) \notin B$. Therefore, $g(h(2 i-1, x)) \cap T \neq \emptyset \wedge f(h(2 i, x)) \cap T \neq \emptyset$, i.e. $g^{\prime}(x) \cap T^{\prime} \neq \emptyset$.

In the case $x \notin A$ it holds for all $i \in\{1, \ldots, k(x)\}$ that $h(2 i-1, x) \in B \Leftrightarrow h(2 i, x) \in B$. Therefore, $g(h(2 i-1, x)) \cap T \neq \emptyset \Leftrightarrow f(h(2 i, x)) \cap T=\emptyset$, which implies that $g^{\prime}(x) \cap T^{\prime}=\emptyset$.

Hence $A$ disjunctively reduces to $T^{\prime}$ via $g^{\prime}$, i.e. $A \in R_{d}^{p}($ TALLY $)$. Since $R_{h d}^{p}(\mathcal{C})$ is closed under complementation for every class $\mathcal{C}$, we get that also $\bar{A}$ disjunctively reduces to some tally set, i.e. $A \in R_{c}^{p}$ (TALLY). Since by Theorem $4.12 \mathrm{IC}[\log$, poly $]=$ $R_{c}^{p}(\mathrm{TALLY}) \cap R_{c}^{p}$ (TALLY) it follows that $A \in \mathrm{IC}[\log$, poly $]$. 


\section{Lowness}

The low and high hierarchies inside NP were introduced by Schöning [Sch83]. The notion of lowness has turned out to be an important structural tool for classifying problems and subclasses of NP not known to be NP-complete or in P. This idea was extended by Balcázar, Book, and Schöning [BBS86] who defined the extended low hierarchy in order to classify decision problems and classes not contained in NP. Allender and Hemachandra [AH92] and Long and Sheu [LS91] refined the extended low hierarchy and proved the optimality of the location of various classes therein.

Definition 5.1 [BBS86, AH92, LS91] The $\Sigma, \Delta$, and $\Theta$ levels of the extended low hierarchy (denoted $E L_{k}^{\Sigma}, E L_{k}^{\Delta}$, and $E L_{k}^{\Theta}$, respectively) are defined as below.

$$
\begin{aligned}
& \text { 1. } E L_{k}^{\Sigma}=\left\{A \mid \Sigma_{k}^{p}(A) \subseteq \Sigma_{k-1}^{p}(\mathrm{SAT} \oplus A)\right\}, k \geq 1 \\
& \text { 2. } E L_{k}^{\Delta}=\left\{A \mid \Delta_{k}^{p}(A) \subseteq \Delta_{k-1}^{p}(\operatorname{SAT} \oplus A)\right\}, k \geq 2 \\
& \text { 3. } E L_{k}^{\Theta}=\left\{A \mid \Theta_{k}^{p}(A) \subseteq \Theta_{k-1}^{p}(\operatorname{SAT} \oplus A)\right\}, k \geq 2
\end{aligned}
$$

Various classes of sets reducible to sparse and tally sets have been shown to be included in the extended low hierarchy (see for example [BBS86, AH92, LS91]). Using the simplicity results of the previous section we are able to derive lowness results for the reduction classes to sparse sets considered here. Some of our extended lowness proofs contain a census argument similar to that used by Hemachandra [Hem87] and Kadin [Kad87]. The following useful lemma gives an upper bound for the complexity of computing the census function of a sparse set.

Lemma 5.2 For every sparse set $S$, census ${ }_{S} \in \mathrm{F} \Theta\left(R_{c}^{n p}(S)\right)$.

Proof The value of census $_{S}\left(1^{n}\right)$ can be computed by a binary search using the oracle set $C:=\left\{\left\langle 0^{n}, m\right\rangle \mid\right.$ there are at least $m$ different strings of length at most $n$ in $\left.S\right\}$ which can easily be seen to be in $R_{c}^{n p}(S)$.

The following technical lemma is used in several of our lowness proofs.

\section{Lemma 5.3}

1. For every set $A$ and $k \geq 2, \Theta_{k}^{p}(\operatorname{SPARSE} \cap \operatorname{NP}(A)) \subseteq \Theta_{2}^{p}\left(\Sigma_{k-2}^{p} \oplus A\right)$.

2. Let $A$ be a set and let $k \geq 3$. If $L$ is in $\Theta_{k}^{p}\left(\operatorname{SPARSE} \cap \mathrm{NP}(A \oplus \mathrm{SAT}) / \mathrm{F}_{2}^{p}(A \oplus\right.$ $\mathrm{SAT})$ ) witnessed by a $\Theta_{k}^{p}$ computation that on input $x$ asks the sparse set only queries of length $p(|x|)$ for some fixed polynomial $p$ then $L$ is in $\Theta_{2}^{p}\left(\Sigma_{k-2}^{p} \oplus A\right)$.

\section{Proof}

1. Let $B$ be a set in $\Theta_{k}^{p}(S)$ for a sparse set $S$ in $\operatorname{NP}(A)$, and let $M$ be a $\Theta$ machine deciding $B$ relative to some oracle $C \in \Sigma_{k-1}^{p}(S)$ that is computed by a $\Sigma_{k-1}^{p}$ oracle machine $M^{\prime}$. Let $p$ be a polynomial bounding the length of the queries to $S$ in the $\Theta_{k}^{p}(S)$ computation of $B$. We outline how $B$ can be computed in $\Theta_{2}^{p}\left(\Sigma_{k-2}^{p} \oplus A\right)$. On input $x$, at first $m=$ census $_{S}\left(1^{p(|x|)}\right)$ is computed (by a $\mathrm{F}_{2}^{p}(A)$ computation, as described in Lemma 5.2). Then machine $M$ is simulated on input $x$ where each 
query $y$ to $C$ is replaced by the query $\langle y, m, p(|x|)\rangle$ to the $\operatorname{NP}\left(\sum_{k-2}^{p} \oplus A\right)$ set $C^{\prime}$ defined as

$$
C^{\prime}=\left\{\langle y, i, j\rangle \mid \exists y_{1}<\cdots<y_{i} \in S^{\leq j}: y \in L\left(M^{\prime},\left\{y_{1}, \ldots, y_{i}\right\}\right)\right\} .
$$

2. Let $B$ be a set in $\Theta_{k}^{p}(S)$ for a sparse set $S$ in $\mathrm{NP}(A \oplus \mathrm{SAT}) / \mathrm{F} \Theta_{2}^{p}(A \oplus \mathrm{SAT})$, and let $M$ be a $\Theta$ machine deciding $B$ relative to some oracle $C \in \Sigma_{k-1}^{p}(S)$ that is computed by a $\Sigma_{k-1}^{p}$ oracle machine $M^{\prime}$. The proof is similar to the one above. The only difference is that now on input $x$ the advice string for instances of $S$ of length $p(|x|)$ and the cardinality of $S^{=p(n)}$ has to be precomputed by an $\mathrm{F}_{2}^{p}(A \oplus \mathrm{SAT})$ computation before the simulation of $M$ on input $x$ is started.

Theorem 5.4 $R_{h d}^{p}\left(R_{c}^{p}(\mathrm{SPARSE})\right) \subseteq E L_{3}^{\Theta}$.

Proof Let $A$ be in $R_{h d}^{p}\left(R_{c}^{p}\right.$ (SPARSE)). Using Theorem 4.2 we can assume that $A \in$ $R_{h d}^{p}\left(R_{c}^{p}(S)\right)$ for a sparse set $S \in \mathrm{NP}(A)$. In order to prove the theorem we have to show that every set $B \in \Theta_{3}^{p}(A)$ is already contained in $\Theta_{2}^{p}(\operatorname{SAT} \oplus A)$. Since $A \in R_{h d}^{p}\left(R_{c}^{p}(S)\right)$, it follows that $B \in \Theta_{3}^{p}(S)$. Since $S$ is in $\mathrm{NP}(A)$ we can apply Lemma 5.3 (part 1 for the case $k=3)$ and obtain that $B$ is in $\Theta_{2}^{p}(\operatorname{SAT} \oplus A)$.

This result is optimal since SPARSE $\nsubseteq E L_{2}^{\Sigma}$ as proved in [AH92]. Item 2 of the following corollary follows from the closure under complementation of the classes in the extended low hierarchy. Item 3 answers an open question in [AH92] and also extends the recent result by Long and Sheu [LS91] that $R_{1-t t}^{p}(\mathrm{SPARSE}) \subseteq E L_{3}^{\Theta}$. Item 4 is a consequence of the closure of the $\Theta$ levels of the extended low hierarchy under $\equiv_{T}^{p}$ [LS91].

\section{Corollary 5.5}

1. $R_{c}^{p}(\mathrm{SPARSE}) \subseteq E L_{3}^{\Theta}$

2. $R_{d}^{p}(\mathrm{co}-\mathrm{SPARSE}) \subseteq E L_{3}^{\Theta}$

3. $R_{b}^{p}(\mathrm{SPARSE}) \subseteq E L_{3}^{\Theta}$

4. $E_{T}^{p}\left(R_{h d}^{p}\left(R_{c}^{p}(\operatorname{SPARSE})\right)\right) \subseteq E L_{3}^{\Theta}$

The following theorem states the generalized $\Theta_{2}^{p}$-lowness of NP(NP $\cap$ SPARSE) $\cap$ $R_{m}^{c o-n p}$ (SPARSE) and is an improvement of Kadin's result that every sparse NP set is low for $\Theta_{2}^{p}$.

Theorem 5.6 If $A \in \mathrm{NP}(\mathrm{NP} \cap \mathrm{SPARSE})$ and $A \leq_{m}^{c o-n p} S$ for a sparse set $S$ then $\Theta_{2}^{p}(A) \subseteq \Theta_{2}^{p}$, i.e. $A$ is low for $\Theta_{2}^{p}$.

Proof Let $A \in \mathrm{NP}\left(S^{\prime}\right)$ for a sparse set $S^{\prime}$ in NP and $A \leq_{m}^{c c^{-n}} S$ for a sparse set $S$. By Theorem 4.9 we can assume that $S \in R_{m}^{n p}(A)$. Since $A \in \operatorname{NP}\left(S^{\prime}\right)$ and since $\mathrm{NP}\left(S^{\prime}\right)$ is closed under $\leq_{m}^{n p}$-reductions it follows that $S \in \mathrm{NP}\left(S^{\prime}\right)$. This shows that $A \in$ co-NP $\left(\operatorname{NP}\left(S^{\prime}\right) \cap \operatorname{SPARSE}\right) \cap \mathrm{NP}\left(S^{\prime}\right)$. Let $S^{\prime \prime} \in \mathrm{NP}\left(S^{\prime}\right) \cap$ SPARSE witness the above inclusion for $A$, i.e. $A \in \operatorname{co-NP}\left(S^{\prime \prime}\right) \cap \mathrm{NP}\left(S^{\prime}\right)$. It is not hard to infer that $\mathrm{NP}(A) \subseteq \mathrm{NP}\left(S^{\prime} \oplus S^{\prime \prime}\right)$. Hence, it follows that $\Theta_{2}^{p}(A) \subseteq \Theta_{2}^{p}\left(S^{\prime} \oplus S^{\prime \prime}\right)$. Since $S^{\prime} \oplus S^{\prime \prime}$ 
is in $\mathrm{NP}\left(S^{\prime}\right) \cap \mathrm{SPARSE}$ we can invoke Lemma 5.3 (part 1 for the case $k=2$ ) to get $\Theta_{2}^{p}\left(S^{\prime} \oplus S^{\prime \prime}\right) \subseteq \Theta_{2}^{p}\left(S^{\prime}\right)$. Since $S^{\prime} \in \mathrm{NP} \cap \mathrm{SPARSE}$, we invoke Lemma 5.3 (part $1, k=2$ ) once more to get $\Theta_{2}^{p}\left(S^{\prime}\right) \subseteq \Theta_{2}^{p}$. Combining the above inclusions yields that $\Theta_{2}^{p}(A) \subseteq \Theta_{2}^{p}$.

The following corollary improves the result of Lozano and Torán [LT91] that every disjunctive self-reducible set that is many-one reducible to some sparse set is low for $\Theta_{2}^{p}$. Since co-NP/log $\subseteq R_{m}^{c o-n p}$ (TALLY) [AKM92] it subsumes all previously known $\Theta_{2}^{p}$-lowness results (e.g. for $\mathrm{NP} \cap R_{m}^{p}$ (SPARSE) and for NP $\cap$ co-NP/log, cf. [LS91]) regarding NP sets reducible to sparse or tally sets.

\section{Corollary 5.7}

1. If $A \in \mathrm{NP} \cap R_{c}^{p}(\mathrm{SPARSE})$ then $\Theta_{2}^{p}(A) \subseteq \Theta_{2}^{p}$.

2. If $A \in \mathrm{NP} \cap R_{m}^{c o-n p}(\operatorname{SPARSE})$, then $\Theta_{2}^{p}(A) \subseteq \Theta_{2}^{p}$.

Although we have simplicity results concerning nondeterministic reduction classes to sparse sets (e.g. Theorem 4.10) extended lowness is not a meaningful measure of lowness for such reduction classes since they contain either NP or co-NP. Nevertheless, the next theorem is a kind of lowness result. We show that the full power of a $\Theta_{3}^{p}$ computation relative to an oracle in $R_{h d}^{p}\left(R_{m}^{c o^{-n p}}\right.$ (SPARSE)) is not needed in order to access the information contained in the oracle.

Theorem 5.8 If $A \in R_{h d}^{p}\left(R_{m}^{c o-n p}(\operatorname{SPARSE})\right)$ then $\Theta_{3}^{p}(A) \subseteq \Theta_{2}^{p}\left(\Sigma_{2}^{p} \oplus A\right)$.

Proof By Theorem 4.10 we can assume that $A$ is in $R_{h d}^{p}\left(R_{m}^{c o-n p}(S)\right)$ for a sparse set $S \in \operatorname{NP}(\operatorname{SAT} \oplus A)$. Therefore, $\Theta_{3}^{p}(A) \subseteq \Theta_{4}^{p}(S) \subseteq \Theta_{4}^{p}(\mathrm{NP}(\mathrm{SAT} \oplus A) \cap \mathrm{SPARSE})$, and by Lemma 5.3 (part 1 for the case $k=4$ ) we get $\Theta_{4}^{p}(\operatorname{NP}(\operatorname{SAT} \oplus A) \cap \operatorname{SPARSE}) \subseteq$ $\Theta_{2}^{p}\left(\Sigma_{2}^{p} \oplus(\mathrm{SAT} \oplus A)\right)$. Now the theorem follows since $\Theta_{2}^{p}\left(\Sigma_{2}^{p} \oplus(\mathrm{SAT} \oplus A)\right) \subseteq \Theta_{2}^{p}\left(\Sigma_{2}^{p} \oplus A\right)$.

Our next lowness result exploits the simplicity of sparse $d$-descriptions for sets in $R_{d}^{p}(\mathrm{SPARSE})$.

\section{Theorem 5.9}

1. $R_{d}^{p}(\mathrm{SPARSE}) \subseteq E L_{3}^{\Theta}$.

2. $R_{1-t t}^{p}\left(R_{d}^{p}(\mathrm{SPARSE})\right) \subseteq E L_{3}^{\Theta}$.

\section{Proof}

1. Let $A$ be in $R_{d}^{p}(\mathrm{SPARSE})$. We have to show that $\Theta_{3}^{p}(A) \subseteq \Theta_{2}^{p}(A \oplus \mathrm{SAT})$. Since $A$ is many-one equivalent to the set $\left\{x 10^{i} \mid i \geq 0 \wedge x \in A\right\}$ we can w.l.o.g. assume that all queries to $A$ in the $\Theta_{3}^{p}(A)$ computation on input $x$ are of length $p(|x|)$ for some fixed polynomial $p$. By Theorem 4.5 it follows that there is a sparse set $S \in \mathrm{NP}(A \oplus \mathrm{SAT}) / \mathrm{F}_{2}^{p}(A)$ such that $A$ is in $R_{d}^{p}(S)$ via some function $f$, where all queries in $f(x)$ are of length $q(|x|)$ for some fixed polynomial $q$. Then it follows by Lemma 5.3 (part 2 for the case $k=3$ ) that $\Theta_{3}^{p}(A) \subseteq \Theta_{3}^{p}(S) \subseteq \Theta_{2}^{p}(A \oplus \mathrm{SAT})$. 
2. Analogously to 1. using Corollary 4.7 instead of Theorem 4.5.

\section{Corollary 5.10}
1. $R_{c}^{p}(\mathrm{co}-\mathrm{SPARSE}) \subseteq E L_{3}^{\Theta}$
2. $E_{T}^{p}\left(R_{d}^{p}(\mathrm{SPARSE})\right) \subseteq E L_{3}^{\Theta}$

Finally we prove the location of IC[log,poly] in the first level of the extended low hierarchy. Since $E L_{1}^{\Sigma}=E L_{2}^{\Theta}=E L_{2}^{\Delta}$ the class IC[log, poly] which equals $R_{c}^{p}$ (SPARSE) $\cap$ $R_{d}^{p}$ (co-SPARSE) is located two levels below $R_{c}^{p}$ (SPARSE) in the extended low hierarchy.

Theorem 5.11 If $A$ has a sparse description that is $\mathrm{P}(\mathrm{SAT} \oplus A)$ printable then $A \in$ $E L_{1}^{\Sigma}$.

Proof Let $S$ be a $\mathrm{P}(\mathrm{SAT} \oplus A)$ printable description for $A$, i.e., $A \in \mathrm{P}(S)$. We have to show that $\mathrm{NP}(A) \subseteq \mathrm{P}(\mathrm{SAT} \oplus A)$. The $\mathrm{NP}(A)$ computation can be simulated by the following $\mathrm{P}(\mathrm{SAT} \oplus A)$ computation: first compute a large enough initial segment of the sparse set $S$ and then simulate the $\mathrm{NP}(A)$ computation where oracle queries to $A$ are answered using the initial segment of $S$.

Since the class APT (almost polynomial time [MP79]) is easily seen to be contained in IC [log, poly] the following corollary subsumes all previously known $E L_{1}^{\Sigma}$-lowness results (e.g. for APT [LS91], for $E_{T}^{p}$ (TALLY) [BB86] and for $R_{m}^{p}$ (TALLY) [AH92]) regarding sets reducible to sparse or tally sets.

\section{Corollary 5.12}
1. $\mathrm{IC}[\log$, poly $] \subseteq E L_{1}^{\Sigma}$
2. $E_{T}^{p}(\mathrm{IC}[\log$, poly $]) \subseteq E L_{1}^{\Sigma}$

Proof Let $A$ be a set in IC[log, poly]. By Theorems 4.14 and 4.12 there is a tally set $T \in \mathrm{P}(\mathrm{SAT} \oplus A)$ such that $A \in R_{c}^{p}(T) \cap R_{d}^{p}(T)$. Since $T$ is actually $\mathrm{P}(\mathrm{SAT} \oplus A)$ printable the $E L_{1}^{\Sigma}$ lowness of $A$ follows by Theorem 5.11.

The following theorem can be proved similarly.

Theorem 5.13 $R_{m}^{n p}(\mathrm{TALLY}) \cap R_{m}^{c o-n p}(\mathrm{TALLY}) \subseteq E L_{1}^{\Sigma}$.

We summarize some of our results on simplicity and lowness in the following table.

\begin{tabular}{|l|l|l|}
\hline For $A$ in the reduction class & simplicity & lowness \\
\hline$R_{h d}^{p}\left(R_{c}^{p}(\mathrm{SPARSE})\right)$ & $\mathrm{NP}(A)$ & $E L_{3}^{\Theta}$ \\
$R_{1-t t}^{p}\left(R_{d}^{p}(\mathrm{SPARSE})\right)$ & $\mathrm{NP}(\mathrm{SAT} \oplus A) / \mathrm{F}_{2}^{p}(A)$ & $E L_{3}^{\Theta}$ \\
$R_{c}^{p}(\mathrm{TALLY}) \cap R_{d}^{p}(\mathrm{TALLY})$ & $\mathrm{P}(\mathrm{SAT} \oplus A)$ & $E L_{1}^{\Sigma}$ \\
$R_{m}^{n p}(\mathrm{TALLY}) \cap R_{m}^{c o-n p}(\mathrm{TALLY})$ & $\mathrm{P}(\mathrm{SAT} \oplus A)$ & $E L_{1}^{\Sigma}$ \\
$R_{m}^{c 0^{-n p}}(\mathrm{SPARSE}) \cap \mathrm{NP} \mathrm{NP} \cap \mathrm{SPARSE}$ & $R_{m}^{n p}(A)$ & $\Theta_{2}^{p}(A) \subseteq \Theta_{2}^{p}$ \\
$R_{h d}^{p}\left(R_{m}^{c 0^{-n p}}(\mathrm{SPARSE})\right)$ & $\mathrm{NP}(\mathrm{SAT} \oplus A)$ & $\Theta_{3}^{p}(A) \subseteq \Theta_{2}^{p}\left(\Sigma_{2}^{p} \oplus A\right)$ \\
\hline
\end{tabular}




\section{Acknowledgments}

The first author is grateful to Uwe Schöning for his hospitality and for the research environment provided at Universität Ulm during the year 1991-92.

\section{References}

[AH92] E. Allender and L. Hemachandra. Lower bounds for the low hierarchy. Journal of the ACM, 39(1):234-250, 1992.

$\left[\mathrm{AHH}^{+} 92\right]$ V. Arvind, Y. Han, L. Hemachandra, J. Köbler, A. Lozano, M. Mundhenk, M. Ogiwara, U. Schöning, R. Silvestri, and T. Thierauf. Reductions to sets of low information content. Proceedings of the 19th International Colloquium on Automata, Languages, and Programming, Lecture Notes in Computer Science, \#623:162-173, Springer Verlag, 1992.

[AHOW] E. Allender, L. Hemachandra, M. Ogiwara, and O. Watanabe. Relating equivalence and reducibility to sparse sets. SIAM Journal on Computing, to appear.

[AKM92] V. Arvind, J. Köbler, and M. Mundhenk. On bounded truth-table, conjunctive, and randomized reductions to sparse sets. To appear in Proceedings 12th Conference on the Foundations of Software Technology 6 Theoretical Computer Science, 1992.

[BB86] J. Balcázar and R. Book. Sets with small generalized Kolmogorov complexity. Acta Informatica, 23(6):679-688, 1986.

[BBS86] J.L. Balcázar, R. Book, and U. Schöning. Sparse sets, lowness and highness. SIAM Journal on Computing, 23:679-688, 1986.

[BDG] J.L. Balcázar, J. Díaz, and J. Gabarró. Structural Complexity I. EATCS Monographs on Theoretical Computer Science, Springer-Verlag, 1988.

[BLS92] H. Buhrman, L. Longpré, and E. Spaan. Sparse reduces conjunctively to tally. Technical Report NU-CCS-92-8, Northeastern University, Boston, 1992.

[GW91] R. Gavaldà and O. Watanabe. On the computational complexity of small descriptions. Proceedings of the 6th Structure in Complexity Theory Conference, 89-101, IEEE Computer Society Press, 1991.

[Hau14] F. Hausdorff. Grundzüge der Mengenlehre. Leipzig, 1914.

[HY84] J. Hartmanis and Y. Yesha. Computation times of NP sets of different densities. Theoretical Computer Science, 34:17-32, 1984.

[Hem87] L. Hemachandra. The strong exponential hierarchy collapses. Proceedings of the 19th ACM Symposium on Theory of Computing, 110-122, 1987. 
[Kad87] J. Kadin. $\mathrm{P}^{\mathrm{NP}[\log n]}$ and sparse Turing-complete sets for NP. Journal of Computer and System Sciences, 39(3):282-298, 1989.

[KL80] R. Karp and R. Lipton. Some connections between nonuniform and uniform complexity classes. Proceedings of the 12th ACM Symposium on Theory of Computing, 302-309, April 1980.

[KoSc85] K. Ko and U. Schöning. On circuit-size complexity and the low hierarchy in NP. SIAM Journal on Computing, 14:41-51, 1985.

[Köb92] J. Köbler. Locating P/poly optimally in the extended low hierarchy. Ulmer Informatik-Bericht 92-05, Universität Ulm, August 1992.

[KSW87] J. Köbler, U. Schöning, and K.W. Wagner. The difference and truth-table hierarchies of NP. Theoretical Informatics and Applications, 21(4):419-435, 1987.

[KT90] J. Köbler and T. Thierauf. Complexity classes with advice. Proceedings 5th Structure in Complexity Theory Conference, 305-315, IEEE Computer Society, 1990.

[Kre89] M.W. Krentel. The complexity of optimization problems. Journal of Computer and System Sciences, 36:490-509, 1988.

[LLS75] R. Ladner, N. Lynch, and A. Selman. A comparison of polynomial time reducibilities. Theoretical Computer Science, 1(2):103-124, 1975.

[LS91] T.J. Long and M.-J. Sheu. A refinement of the low and high hierarchies. Technical Report OSU-CISRC-2/91-TR6, The Ohio State University, 1991.

[LT91] A. Lozano and J. Torán. Self-reducible sets of small density. Mathematical Systems Theory, 24:83-100, 1991.

[Mah82] S. Mahaney. Sparse complete sets for NP: Solution of a conjecture of Berman and Hartmanis. Journal of Computer and System Sciences, 25(2):130-143, 1982.

[MP79] A. Meyer, M. Paterson. With what frequency are apparently intractable problems difficult? Tech. Report MIT/LCS/TM-126, Lab. for Computer Science, MIT, Cambridge, 1979.

[OKSW] P. Orponen, K. Ko, U. Schöning, and O. Watanabe. Instance complexity. Journal of the ACM, to appear.

[OW91] M. Ogiwara and O. Watanabe. On polynomial-time bounded truth-table reducibility of NP sets to sparse sets. SIAM Journal on Computing, 20(3):471483, 1991.

[Sch83] U. Schöning. A low hierarchy within NP. Journal of Computer and System Sciences, 27:14-28, 1983.

[Sch86] U. Schöning. Complexity and Structure, Lecture Notes in Computer Science, \#211, Springer-Verlag, 1985 
[SL92] M.-J. Sheu and T.J. Long. The extended low hierarchy is an infinite hierarchy. Proceedings of 9th Symposium on Theoretical Aspects of Computer Science, Lecture Notes in Computer Science, \#577:187-189, Springer-Verlag 1992.

[TB91] S. Tang and R. Book. Reducibilities on tally and sparse sets. Theoretical Informatics and Applications, 25:293-302, 1991.

[Wag87] K.W. Wagner. More complicated questions about maxima and minima, and some closures of NP. Theoretical Computer Science, 51:53-80, 1987.

[Wag90] K.W. Wagner. Bounded query classes. SIAM Journal on Computing, 19(5):833-846, 1990. 